\title{
Power Smart In-door Optical Wireless Link Design
}

\section{P. J. Marraccini}

\author{
N. A. Riza \\ n.riza@ucc.ie
}

\begin{abstract}
Department of Electrical and Electronic Engineering, University College Cork, College Road, Cork, Ireland

Department of Electrical and Electronic Engineering and Tyndall National Institute, University College Cork, College Road, Cork, Ireland
\end{abstract}

Presented for the first time, to the best of the authors' knowledge, is the design of a power smart in-door optical wireless link that provides lossless beam propagation between Transmitter $(T)$ and Receiver $(R)$ for changing link distances. Each $T / R$ unit uses a combination of fixed and variable focal length optics to smartly adjust the laser beam propagation parameters of minimum beam waist size and its location to produce the optimal zero propagation loss coupling condition at the $\mathrm{R}$ for that link distance. An Electronically Controlled Variable Focus Lens (ECVFL) is used to form the wide field-of-view search beam and change the beam size at $R$ to form a low loss beam. The T/R unit can also deploy camera optics and thermal energy harvesting electronics to improve link operational smartness and efficiency. To demonstrate the principles of the beam conditioned low loss indoor link, a visible $633 \mathrm{~nm}$ laser link using an electro-wetting technology liquid ECVFL is demonstrated for a variable 1 to $4 \mathrm{~m}$ link range. Measurements indicate a 53\% improvement over an unconditioned laser link at $4 \mathrm{~m}$. Applications for this power efficient wireless link includes mobile computer platform communications and agile server rack interconnections in data centres. [DOI: http://dx.doi.org/10.2971/jeos.2011.11054]

Keywords: green optical wireless, low power wireless network, indoor wireless, free space optical communication, optical transmitter

\section{INTRODUCTION}

It was realized in the late 1970s that diffused Infrared (IR) light much like Radio Frequency (RF) radiation filling a room could be used for wireless data communications [1]. This indoor optical wireless technique is also known as the Diffused Infrared Radiation (DFIR) and is very effective in preventing physical blocking of light to the receiver and tolerant to transmitter/receiver mobility. Since this method uses scattered light in room, it is highly power inefficient and also limits achievable data rates due to multipath effects. To counter these limitations of the DFIR indoor wireless method, highly directional light beam communication, also known as Line-ofSight (LOS) infrared communications or the Directed-beam IR (DBIR) technique, was proposed and demonstrated [2, 3]. Today, wireless optical communications is still an active area of research $[4,6]$.

The use of agile spatially reconfigurable laser beams has been previously proposed for space (i.e., inter-satellite) [7] and indoor wireless applications [8]. Specifically, reference 8 proposed a new hybrid indoor optical wireless method, now called Hybrid Diffused-LOS, as it is a combination of the DFIR and DBIR methods. Earlier, a smart free-space link design was proposed and theoretically analysed for a variety of link ranges varying from short indoor applications to long distance inter-satellite links [9]. In particular, these smart link designs use Transmitter (T) and Receiver (R) electronically agile beam conditioning lens optics that adjust focus based on specific link distance requirements for a given scenario. Within the framework of paraxial optics and beam forming optics de- vice limits, one is able to produce a zero propagation link coupling loss design for a given range of link distances. Recent advances in micro-devices, such as laser modules, micro-lenses, Electronically Controlled Variable Focus Lenses (ECVFLs), and Micro-Electro-Mechanical Systems (MEMS) beam directing micro-mirrors, points to the realization of a new higher compactness micro-optics scale optical wireless $\mathrm{T}$ and $\mathrm{R}$ modules that can be readily adapted for use in a smart indoor low loss application link design. For example, the emergence of low cost visible light laser pointers and laser scanning based colour projection displays points to the applicability of low power visible laser sources for indoor optical wireless link applications. Note that presently, visible light-based indoor wireless communications is considered to be an excellent alternative to IR-based optical wireless, given that visible light usage has benefits such as low cost sources, e.g., white light emission Light Emitting Diodes (LEDs) [10, 12], smaller T and $\mathrm{R}$ apertures via use of shorter wavelengths and easier beam alignment due to human and visible CCD camera observation of free-space beams. In addition, low cost visible laser pointers have become available with good beam divergence and beam shape properties, these can be exploited for short range indoor optical wireless links such as the one attempted in this paper. Nevertheless, one must be careful about eye safety issues when using visible laser-pointer style sources. Hence visible laser power levels should be restricted to low power safe levels such as done in laser scanning pico-display applications [13]. Given that proposed smart optical link design provides the lowest propagation loss between a transmitter and 
receiver, the lowest laser power needed can definitely be used for a particular bit rate, thus helping the visible light eye safety issue.

Hence, this paper will focus on visible laser pointer-like sources for link design, although other eye safe band (e.g., $780 \mathrm{~nm}, 850 \mathrm{~nm}, 1300 \mathrm{~nm}$, and $1550 \mathrm{~nm}$ ) laser sources can also be applied to the proposed smart link concept. The key innovation proposed in this paper is the use of the ECVFL to adjust beam parameters for low loss operations with changing link distances as well as use of the ECVFL controlled wide area search beam to initially align the transmitter with the receiver. The rest of the paper describes the smart link design and a basic visible light proof-of-principle link design experiment.

\section{PROPOSED SMART OPTICAL WIRELESS LIN K}

Figure 1 shows the proposed compact and power smart optical wireless link design using two independent transceiver or $\mathrm{T} / \mathrm{R}$ units. Figure 1(a) link design allows simultaneous $\mathrm{T}$ and $\mathrm{R}$ communications via a common $\mathrm{T} / \mathrm{R}$ aperture that uses a FSO circulator device to physically separate the $\mathrm{T}$ and $\mathrm{R}$ beam paths in each $T / R$ unit. The transmit data signal in Figure 1 electrically modulates the laser module. The received data signal is produced by the photodetector in the T/R unit. The T and $R$ beams are spatially conditioned by the concave lens and the ECVFL in the T/R unit. The concave lens (also called the Negative Lens or NL) of focal length $f_{N L}$ is used to produce beam expansion of the Gaussian beam from the laser module so that the laser light completely illuminates the ECVFL aperture. Typically, the aperture of the ECVFL and other microoptics in the $\mathrm{T} / \mathrm{R}$ unit do not exceed a $5 \mathrm{~mm}$ diameter, giving a pencil-like compact design to the proposed $T / R$ units. The ECFVLs focal length $F$, is controlled by an electrical signal (e.g., voltage level or drive frequency) which in-turn controls the minimum beam waist diameter $2 w_{M i n}$ and its position $d_{M i n}$ with respect to the transmitter beam ECVFL location. Depending on the link distance $L, F$ can be controlled to produce the desired Gaussian beam propagation such that it exhibits the self-imaging effect between the two communicating $T / R$ units and thus forms a zero propagation loss link. Other notations for Figure 1 are as follows: $w_{0}$ : minimum beam waist radius of the laser; $w_{E L}$ : beam waist radius at the transmit ECVFL; $w_{E L 2}$ : beam waist radius after the receive $\mathrm{ECVFL} ; w_{R}$ : beam waist radius at the receive ECVFL; $w_{P D 1}$ : beam waist at Photo-Detector 1 (or PD1); $w_{P D 2}$ : beam waist at Photo-Detector 2 (or PD2); $d_{c}$ : distance from $2 w_{0}$ position to optical circulator; $d_{N L C}$ : distance from optical circulator position to NL; $d_{N L}$ : distance from $2 w_{0}$ position to NL; $d_{S}$ : distance from NL to the ECVFL; $d_{M i n}$ : distance from the ECVFL to the $2 w_{\text {Min }}$ position; $d_{R}$ : distance from $2 w_{\text {Min }}$ location to the $\mathrm{T} / \mathrm{R}$ unit receiving the ECVFL conditioned laser beam; $\mathrm{D}$ : diameter of ECVFL aperture. For a symmetrical link providing the designed lossless beam propagation operations, $d_{R}=d_{\text {Min }}$. The Figure 1(a) link can also be operated in an asymmetrical design where $d_{R}$ is not equal to $d_{M i n}$. The asymmetrical case where $d_{R}>d_{\text {Min }}$ implies that the beam diameter at the receiving ECVFL lens is larger than the ECVFL diameter at the distant $\mathrm{T} / \mathrm{R}$ unit; therefore the zero loss condition will not be met

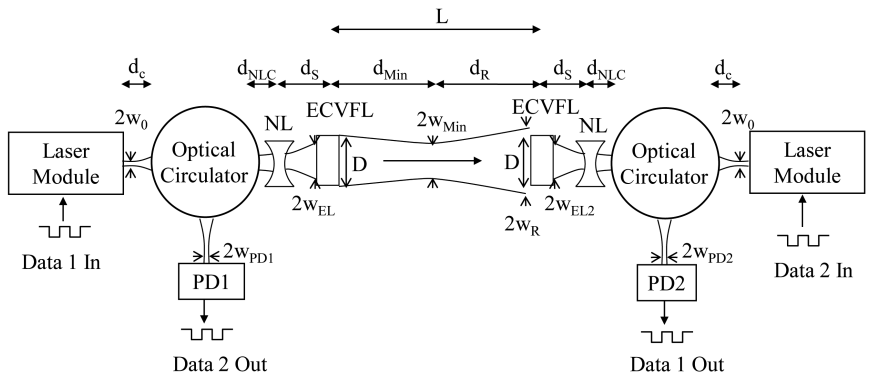

FIC. 1a The proposed smart wireless optical data communication link designs. Common Transmit $(T)$ and Receive ( $R$ ) Aperture smart T/R module design.

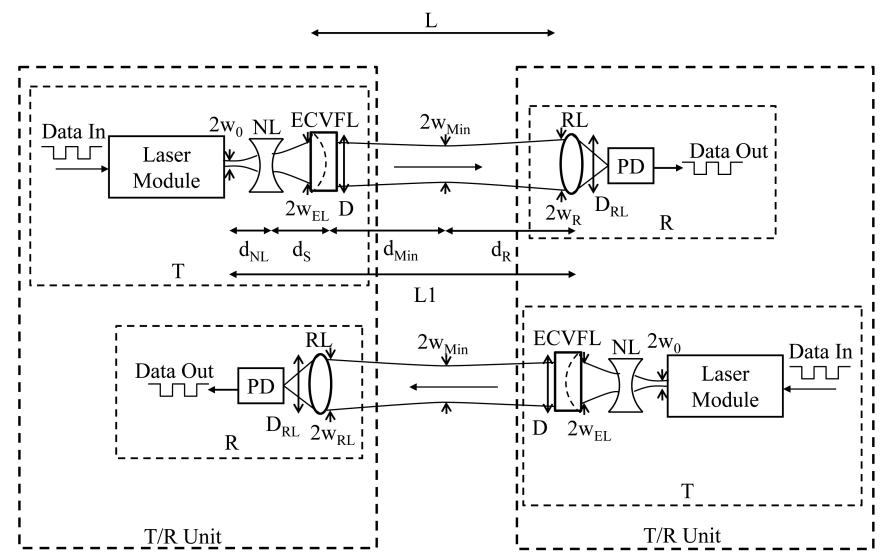

FIG. 16 The proposed smart wireless optical data communication link designs. Independent $\mathrm{T}$ and $\mathrm{R}$ apertures smart $\mathrm{T} / \mathrm{R}$ module design.

leading to incrementally higher light propagation loss as $d_{R}$ exceeds $d_{\text {Min }}$. Figure 1(b) shows an alternate link design with two $\mathrm{T} / \mathrm{R}$ units communicating with physically separate $\mathrm{T}$ and $\mathrm{R}$ optical apertures. Due to this effect each $\mathrm{T} / \mathrm{R}$ unit contains physically separate transmitter and receiver modules. $D_{R L}$ is the diameter of the receiver capture lens RL. The Figure 1(b) $\mathrm{T} / \mathrm{R}$ unit design is suitable when high optical isolation $(>25$ $\mathrm{dB}$ ) is required between the $\mathrm{T}$ and $\mathrm{R}$ channels and when during an asymmetrical link operation, additional receive light capture is desired via a receive lens aperture size that exceeds the typical small ECVFL diameter.

The smart link designs can be analysed using ABCD matrix analysis of Gaussian beams. The results of this analysis are presented here, additionally readers can refer to [14] for details on the derivations. Using ABCD analysis for the setup of Figure 1(b), the beam waist at any location is given as:

$$
w(z)=\sqrt{\frac{\lambda}{\pi} \frac{\left(A z_{0}\right)^{2}+B^{2}}{z_{0}}} .
$$

where $\lambda$ is the wavelength of light, $z_{0}$ is the Rayleigh range of the laser and $A$ and $B$ are elements of the ABCD matrix. To find $w_{E L}$, Eq. (1) is used with the ABCD matrix that describes the laser beam propagation to that point where:

$$
\left[\begin{array}{ll}
A & B \\
C & D
\end{array}\right]=\left[\begin{array}{cc}
1-\frac{d_{S}}{f_{N L}} & d_{S}+d_{N L}-\frac{d_{S} d_{N L}}{f_{N L}} \\
-\frac{1}{f_{N L}} & 1-\frac{d_{N L}}{f_{N L}}
\end{array}\right]
$$

When a minimum beam waist is formed, the real part of the qparamteter is zero. Using ABCD Gaussian beam optics leads 
to:

$$
A C z_{0}^{2}+B D=0 .
$$

Now substituting the appropriate ABCD elements for Figure 1(b) gives:

$$
a F^{2}+b F+c=0,
$$

where:

$$
\begin{aligned}
a= & {\left[z_{0}^{2}\left(d_{M i n}+d_{S}-f_{N L}\right)+d_{N L}^{2}\left(d_{S}+d_{M i n}-f_{N L}\right)\right.} \\
& \left.+f_{N L}^{2}\left(d_{N L}+d_{S}+d_{M i n}\right)-2 d_{N L} f_{N L}\left(d_{S}+d_{M i n}\right)\right] \\
b= & {\left[z_{0}^{2}\left(2\left[f_{N L}\left(d_{S}+d_{M i n}\right)-d_{S} d_{M i n}\right]-d_{S}^{2}-f_{N L}^{2}\right)\right.} \\
& +2 d_{N L}^{2}\left(d_{S} f_{N L}+f_{N L} d_{M i n}-d_{S} d_{M i n}\right) \\
& -2 f_{N L}^{2}\left(d_{N L} d_{S}+d_{N L} d_{M i n}+d_{S} d_{M i n}\right)+4 f_{N L} d_{N L} d_{S} d_{M i n} \\
& \left.+2 d_{N L} d_{S}^{2} f_{N L}-f_{N L}^{2}\left(d_{N L}^{2}+d_{S}^{2}\right)-d_{N L}^{2} d_{S}^{2}\right] \\
c= & {\left[d_{M i n} z_{0}^{2}\left(d_{S}^{2}+f_{N L}^{2}-2 d_{S} f_{N L}\right)\right.} \\
& +2 d_{N L} d_{S} d_{M i n}\left(f_{N L}^{2}-f_{N L} d_{N L}-f_{N L} d_{S}\right) \\
& \left.+\left(d_{N L} d_{S}\right)^{2} d_{M i n}+f_{N L}^{2} d_{M i n}\left(d_{N L}^{2}+d_{S}^{2}\right)\right]
\end{aligned}
$$

Note that larger input beam sizes allow for a larger achievable link distance.

Now the T/R unit link design parameters $f_{N L}, d_{N L}, d_{S}$, and $d_{\text {Min }}$ should be chosen such that the beam radius $w_{E L}$ described by Eq. (1) is smaller than the radius of the ECVFL. Then Eq. (4) can be solved to give:

$$
F=\frac{-b \pm \sqrt{b^{2}-4 a c}}{2 a}
$$

Since $F$ should be real, $b^{2} \geq 4 a c$ for a valid link design. The choice of $F$ will determine the size of the minimum beam waist along with the other system parameters. Note that the focal length of all lenses in the system must satisfy $\mathrm{F} \# \geq 1.67$ [9] since this analysis is done within the paraxial approximation. Using the two solutions obtained for $F$, one solves for the minimum beam waist $w_{\text {Min }}$ as follows:

$$
\begin{aligned}
w_{\text {Min }} & =\sqrt{\frac{\lambda}{\pi} \frac{z_{0}}{\left(C z_{0}\right)^{2}+D^{2}}} \\
{\left[\begin{array}{cc}
A & B \\
C & D
\end{array}\right]=} & {\left[\begin{array}{cc}
1 & d_{\text {Min }} \\
0 & 1
\end{array}\right]\left[\begin{array}{cc}
1 & 0 \\
-\frac{1}{F} & 1
\end{array}\right]\left[\begin{array}{cc}
1 & d_{S} \\
0 & 1
\end{array}\right] } \\
& {\left[\begin{array}{cc}
1 & 0 \\
-\frac{1}{f_{N L}} & 1
\end{array}\right]\left[\begin{array}{cc}
1 & d_{N L} \\
0 & 1
\end{array}\right], }
\end{aligned}
$$

The size of $w_{M i n}$ should be real and less than $w_{E L}$. Since the purpose of the proposed communication link is to achieve low optical power loss, the solution for $F$ that gives the lowest transmit aperture beam divergence should be used. Although both $F$ solutions have the same loss in the symmetric link case, having a smaller beam divergence reduces the link loss for distances of $d_{R}>d_{M i n}$. Since it is known that the divergence is inversely proportional to the minimum beam waist [14] the $F$ solution that gives the larger $w_{\text {Min }}$ minimum beam waist value will give the smaller transmit beam divergence and thus a reduced power loss if $d_{R}>d_{\text {Min }}$. In order to find the beam waist radius at the receiving lens, the ABCD analysis is applied from $w_{0}$ to $w_{R}$ for Figure 1(b) giving:

$$
\begin{aligned}
{\left[\begin{array}{cc}
A & B \\
C & D
\end{array}\right]=} & {\left[\begin{array}{ll}
1 & L \\
0 & 1
\end{array}\right]\left[\begin{array}{cc}
1 & 0 \\
-\frac{1}{F} & 1
\end{array}\right]\left[\begin{array}{cc}
1 & d_{S} \\
0 & 1
\end{array}\right] } \\
& {\left[\begin{array}{cc}
1 & 0 \\
-\frac{1}{f_{N L}} & 1
\end{array}\right]\left[\begin{array}{cc}
1 & d_{N L} \\
0 & 1
\end{array}\right] }
\end{aligned}
$$

Substituting the elements of Eq. (10) into Eq. (1) gives $w_{R}$. For low smart link propagation loss, this beam waist radius $w_{R}$ should be smaller than the diameter of receiving lens. For the case of a symmetric link, the beam waist at the output of the transmitting ECVFL and the size of the beam at the receiving lens should be the same. Similarly, the equations for Figure 1(a) could be derived given the ABCD matrix of the optical circulator used. The smart link beam propagation can be compared to unconditioned Gaussian beam propagation over the distance $L 1$, where $L 1=d_{N L}+d_{S}+L$. The beam waist for the unconditioned Gaussian beam is given by [14]:

$$
w_{N}(z)=w_{0} \sqrt{1+\left(\frac{L 1}{z_{0}}\right)^{2}} .
$$

Now that the beam waist at the receiver location for the smart link and unconditioned Gaussian beam propagation-based link are known, the advantage of the proposed smart link design in terms of optical power at the receiving aperture of the $\mathrm{T} / \mathrm{R}$ unit can be analysed. For a Gaussian beam, the receive aperture collected optical power is given by integrating the irradiance over the area of the receiving aperture giving:

$$
\begin{aligned}
P(z) & =\int_{0}^{2 \pi} \int_{0}^{a} I_{0} \frac{w_{0}^{2}}{w^{2}(z)} e^{-\frac{2 r^{2}}{w^{2}(z)}} r d r d \phi \\
& =2 \pi \int_{0}^{2 \pi} I_{0} \frac{w_{0}^{2}}{w^{2}(z)} e^{-\frac{2 r^{2}}{w^{2}(z)}} r d r .
\end{aligned}
$$

where $I_{0}$ is the laser beam peak intensity at the $w_{0}$ location at the exit of the laser module, $w(z)$ is the beam waist at a specific location given by substituting Eq. (9) into Eq. (1) for the smart link and by Eq. (10) for the unconditioned Gaussian beam, and $a$ is the radius of the receiving aperture, i.e., the receiving lens in the $T / R$ unit. To compare the results of the smart link and the unconditioned Gaussian beam propagation link, Eq. (11) can be normalized as follows:

$$
P_{\text {Norm }}(z)=\frac{\int_{0}^{a} e^{-\frac{22^{2}}{w^{2}(z)}} r d r}{\int_{0}^{\infty} e^{-\frac{2 r^{2}}{w^{2}(z)}} r d r} .
$$

Hence, the normalized percentage optical power loss for the FSO link is given by:

$$
P_{\text {Loss }}(z)=100\left[1-P_{\text {Norm }}(z)\right]
$$

\section{EXPERIMENTAL DEMONSTRATION}

Using the Figure 1(b) link design, a proof of concept demonstration of the smart indoor optical wireless link design is implemented for a visible laser pointer-type source. Since the focus of the paper is optical propagation loss reduced link design, an unmodulated laser is deployed. It is well known that 
direct or indirect modulation of todays visible laser diodes is readily possible for high ( $>100 \mathrm{Mbps}$ ) data rates. Today, a typical laser pointer module for red light has a beam divergence half-angle of $0.6 \mathrm{mrad}$ and a Gaussian minimum beam waist radius $w_{0}$ of $0.5 \mathrm{~mm}$. The visible source deployed has similar characteristics and is a Melles Griot Model 05-LHP-991 $10 \mathrm{~mW}$ He-Ne laser with $\lambda=632.8 \mathrm{~nm}$ a beam divergence of $0.62 \mathrm{mrad}$ and $1 / e^{2}$ beam radius $w_{0}$, of $0.325 \mathrm{~mm}$. Specifically, for the experiment a symmetric (i.e., $w_{E L}=w_{R L}$ ) agile link is designed with a typical indoor room range of $L=4.01 \mathrm{~m}$ where $L=L 1-\left(d_{N L}+d_{S}\right)$. The ECVFL used in the link is a Varioptic (France) Aortic Model 320 liquid lens with a D = $3 \mathrm{~mm}$ aperture. This ECVFL is a broadband visible light variable focus lens that uses electrowetting technology. Expansion of the laser module beam waist for higher beam collimation is implemented using the concave lens (or NL) that has a $f_{N L}=-$ $15 \mathrm{~cm}$. Since the diameter of the ECVFL is $3 \mathrm{~mm}$, the NL and ECVFL are placed with a $d_{N L}=16.55 \mathrm{~cm}$ and $d_{S}=23 \mathrm{~cm}$ such that the $1 / e^{2}$ beam diameter at the ECVFL input plane is less than the ECVFL aperture. Specifically, the $1 / e^{2}$ beam diameter $2 w_{E L}$ at the ECVFL input plane is chosen to satisfy $\mathrm{D}=1.67 \times 2 w_{E L}$ that corresponds to the ECVFL $3 \mathrm{~mm}$ diameter aperture capturing $99.6 \%$ of input light intensity with a theoretical $w_{E L}=899.5 \mu \mathrm{m}$. Using link design Eq. (4) and solving for $d_{\text {Min }}$ gives $L=0.1 \mathrm{~m}$ for the shortest positive ECVFL focal length $F=4.4 \mathrm{~cm}$. Using link design Eq. (4) and solving for $F$ for a desired link distance $L=4.1 \mathrm{~m}$ gives $F=33.44 \mathrm{~cm}$ for the ECVFL. Note that the deployed ECVFL can be electronically controlled from a negative lens with $F=-19.9 \mathrm{~cm}$ to a positive lens with a range of $F=4.4 \mathrm{~cm}$ to $F=40 \mathrm{~cm}$. In the experiment, $F$ is controlled by varying the duty cycle from $82.2 \%$ to $84.4 \%$ for the $40 \mathrm{kHz}$ frequency pulsed wave drive signal. For example, the ECVFL focal lengths are $36.62 \mathrm{~cm}$, $36.3 \mathrm{~cm}$, and $33.4 \mathrm{~cm}$ for duty cycles of $82.4 \%, 82.64 \%$, and $83.12 \%$, respectively. As a moving wireless terminal or agile FSO link connection changes the deployed link distance, the ECFVL $F$ is changed to maintain the lossless link propagation self-imaging condition between the transmit and receive optical terminals. In order to test this smart link idea, a receiver in the form of a laser beam profiler is positioned at the changing $L$ distances to record the receive plane incident laser beam size. Using a highly accurate Digital Micromirror Device (DMD) based beam profiler [15], one can accurately measure the Gaussian beam size and then compute the captured power using Eq. (11) for a given receive lens aperture. In addition, to visualise the effect of the different beam sizes on the receiver plane, a standard visible light CCD camera is used to observe the smart link agile beam.

The percentage optical power loss $P_{\text {Loss }}$ due to beam propagation for the wireless optical link is given by Eq. (13). Figure 2 shows the designed percentage optical power loss for the two types of links. Figure 2 was produced using Eqs. (12) and (13) with Eq. (9) substituted into Eq. (1) for the smart link and Eq. (10) for the non-smart link conditions. Note that given the deployed ECVFL has a D $=3 \mathrm{~mm}$, the receive aperture radius used for the link receiver lens is set to $a=\mathrm{D} / 2=1.5 \mathrm{~mm}$ for Eq. (12). The design plots assume zero transmission and reflection loss for the NL and ECVFL given that these optics can be Anti-Reflection (AR) coated for the laser wavelength. Results from the conducted experiment with non-AR coated

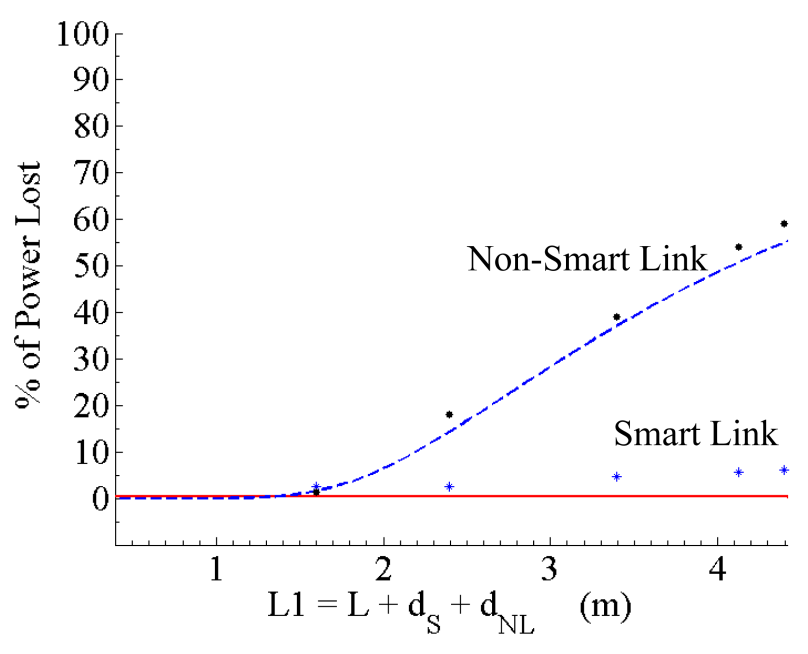

FIC. 2a Shown are the designed and measured optical loss percentage values for the non-smart and smart wireless optical links operating within the designed $4 \mathrm{~m}$ agile self -imaging range of the link. The design assumes a $3 \mathrm{~mm}$ diameter receive lens that matches the $3 \mathrm{~mm}$ diameter of the transmit ECVFL.

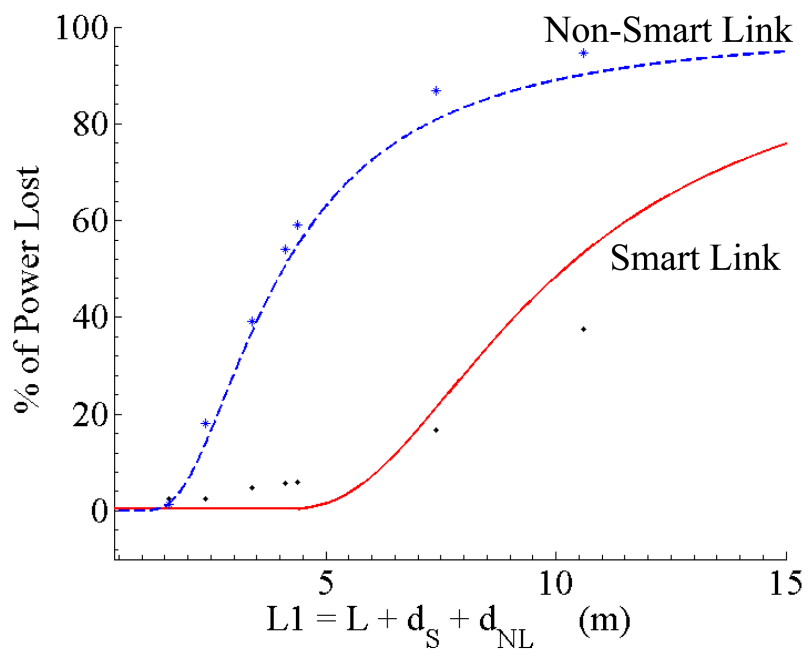

FIG. $2 b$ Shows the designed and measured optical loss percentage values for the nonsmart and smart wireless optical links operating outside the designed $4 \mathrm{~m}$ agile self -imaging range of the link.

optical elements shows that the optical losses were $10 \%$ and $7 \%$ for the NL and ECVFL, respectively. In order to obtain the received optical power measurement, a $3 \mathrm{~mm}$ diameter iris is placed just before the $1 \mathrm{~cm}^{2}$ active area receive photodetector (Newport 918D-UV) to make an aperture diameter equivalent to the ECVFL aperture. Data points on the Figure 2 plots are shown assuming zero-loss components. Figure 2(a) shows that for the changing link distance $L$ from $1 \mathrm{~m}$ to $4 \mathrm{~m}$, the experimental smart link provides a low $<7 \%$ optical percentage loss compared to the non-smart link with an experimental $59.07 \%$ and theoretical $55.2 \%$ loss at $L=4 \mathrm{~m}$. Thus a $53.04 \%$ improvement over the non-smart link was experimentally observed at $L=4 \mathrm{~m}$. Ideally, the smart link loss should be zero, but experimental non-ideal behaviour of the Varioptic ECVFL (e.g., aberrations, coma, etc) and laser beam Gaussian quality restrict the link performance to meet ideal design theory. Improvements in demonstrated link loss can be achieved by using higher optical and beam quality optics (i.e., laser and ECVFL) for the smart link. Given that link distances could ex- 
ceed the designed $4 \mathrm{~m}$ smart link distance, Figure 2(b) shows the designed and measured optical loss percentage values for the non-smart and smart wireless optical links operating outside the designed $4 \mathrm{~m}$ agile self-imaging range of the link. The low loss benefits of the smart link are clear and shown for link distances up-to $15 \mathrm{~m}$. Data shows that at $15 \mathrm{~m}$, the non-smart link percentage loss is $92.8 \%$ versus the $61.5 \%$ for the smart link. In the smart link case, $F$ is set for a $4 \mathrm{~m}$ link distance.

Additional range symmetric links can be designed where the link range is defined between the $L$ distance limits of $L_{M i n} \leq$ $L \leq L_{\text {Max }}$. For example, a goal can be to design a link with $5 \mathrm{~m} \leq L \leq 10 \mathrm{~m}$ range using hardware such a laser module with $\lambda=632.8 \mathrm{~nm}$ and $w_{0}=500 \mu \mathrm{m}$, and a NL with $f_{N L}=-10 \mathrm{~cm}$. For the T/R module design, $d_{N L}$ should be less than the Rayleigh range $z_{0}$ as then the choice of $d_{N L}$ has a minimal effect on $w_{E L}$. In order to preserve the compact design, $d_{N L}$ is chosen to be $1 \mathrm{~cm}$. An initial estimate for $w_{E L}$ is made by using $w_{E L}=\sqrt{\lambda L_{M a x} / \pi}$; this leads to an initial estimate of $w_{E L}=1.42 \mathrm{~mm}$ for $10 \mathrm{~m}$ link range. Using Eq. (2) to solve Eq. (1) for an initial estimate of $d_{S}$ it is found to be $27.06 \mathrm{~cm}$. The calculated value of $d_{S}$ along with the other design parameters and $d_{\text {Min }}=L_{M a x} / 2=5 \mathrm{~m}$ are substituted into Eqs. (5) and (6) to provide a value for $F$ that in this case comes out to be imaginary. As $F$ was an imaginary value, increasing the estimate for $d_{S}$ to $27.43 \mathrm{~cm}$ results in the value $F$ to become positive and equal to $F=40.61 \mathrm{~cm}$ or $F=40.51 \mathrm{~cm}$. Alternatively if a positive value for $d_{S}$ is obtained, one can decrease $d_{S}$ till it cannot be decreased further without $F$ becoming imaginary. Note that the ECVFL chosen must satisfy the condition $2 w_{E L}<$ D. Solving Eqs. (4) and (6) with $d_{M i n}=L_{M i n} / 2=2.5 \mathrm{~m}$ gives $F=41.8 \mathrm{~cm}$ or $F=36.51 \mathrm{~cm}$. Using Eqs. (7) and (8), the $w_{\text {Min }}$ for each focal length pair can be compared. Note that though both solutions of $F$ are valid, the solutions of $F$ that give the larger $w_{M i n}$ (via the longer $F$ value) are chosen as they have less divergence and thus a smaller beam waist when $d_{R}$ $>d_{\text {Min }}$ allowing for low loss link operations when $L>L_{\text {Max }}$. Hence, the ECVFL for the designed $5 \mathrm{~m}$ to $10 \mathrm{~m}$ link requires operations with $40.61 \mathrm{~cm} \leq F \leq 41.8 \mathrm{~cm}$, an achievable ECVFL device performance. The length of the example smart transmit module is given by $d_{N L}+d_{S}=27.06 \mathrm{~cm}+1 \mathrm{~cm}=28.06 \mathrm{~cm}$. In comparison, when using the reference [9] low loss wireless link module design for the same design specifications, i.e., $L_{M i n}=5 \mathrm{~m}, L_{M a x}=10 \mathrm{~m}, w_{E L}=1.42 \mathrm{~mm}, \lambda=632.8 \mathrm{~nm}$, $w_{0}=500 \mu \mathrm{m}$, a fixed focal length convex collimating lens of $3.52 \mathrm{~m}$ focal length is required to give equivalent values of $d_{N L}+d_{S}=3.52 \mathrm{~m}+3.52 \mathrm{~m}=7.04 \mathrm{~m}$ with the ECVFL focal length variation from $2.68 \mathrm{~m} \leq F \leq 10 \mathrm{~m}$. The prior reference 9 module design for a typical visible laser pointer-like source fed low loss link results in a much longer module size in addition to requiring an ECVFL with a larger dynamic range for focal length control. The proposed module design in Figure 1 is highly compact and deploys highly engineered micro-optic components for optimal low loss wireless link designs. The $\mathrm{T} / \mathrm{R}$ module design in [9] is more suited for Single-Mode Fibre (SMF)-to-freespace coupled light transmission and detection within the module as it uses the much smaller SMF core size $w_{0}$ values like $2 w_{0}=9 \mu \mathrm{m}$ for the $1550 \mathrm{~nm}$ near IR wavelength typical for long distance telecommunications fibre networks.

An important feature of the proposed smart link is its ability

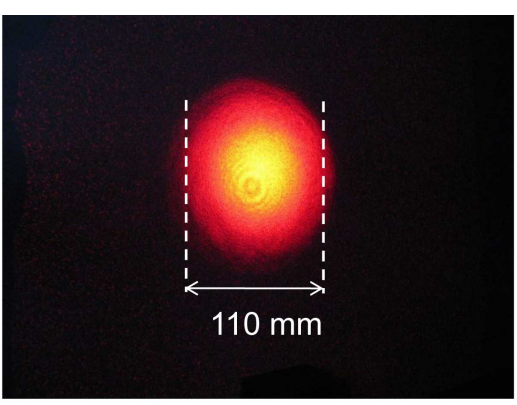

(a)

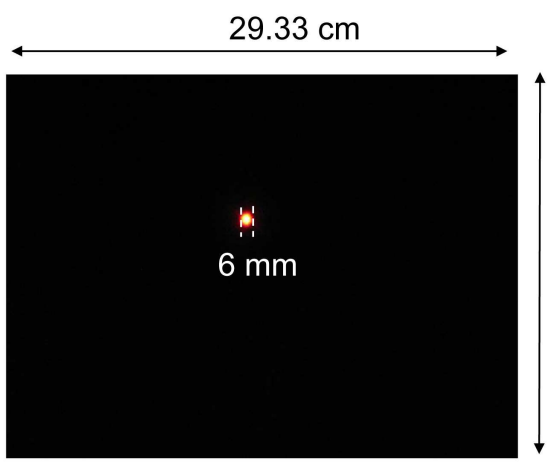

$39.11 \mathrm{~cm}$

(b)

FIG. 3 Shown are CCD camera images (at uniform same scale) of the smart link operating in its wide area search beam mode for a link distance parameter of $\mathrm{L} 1=280.5 \mathrm{~cm}$. (a) Search beam shown using ECVFL F $=4.4 \mathrm{~cm}$. (b) Non-smart link receive beam.

to provide a wide area big search beam to establish a search and hand-shake operation with another remote receiving $T / R$ unit. The search mode operation can be achieved by operating the ECFVL as a short focus convex lens so as to spread out the transmit beam into a wider zone to locate a possible $T / R$ unit. Upon establishing an initial detection with a remote $T / R$ unit, the ECVFL focal length is brought to a longer convex focal length for optimal low loss smart link operations. Figure 3 shows CCD camera images (at uniform scale) of the smart link operating in its wide area search beam mode for a link distance parameter of $L 1=280.5 \mathrm{~cm}$. Figure $3(\mathrm{a})$ shows the $11 \mathrm{~cm}$ diameter search beam obtained using ECVFL $F=4.4 \mathrm{~cm}$. and Figure 3(b) shows the non-smart link received beam. The Figure 3 data indicates that using the present liquid lens ECVFL, a search beam that is approximately 18 times bigger in diameter than the unconditioned non-smart link beam can be generated giving excellent beam search capabilities. In addition, the expanded search beam mode can be used to create spatially controlled cells for optical illumination in an indoor wireless scenario.

\section{CONCLUSION}

In conclusion, a power smart optical wireless link design is presented for low loss indoor operations with changing link distances. The smart link design uses fixed focal length optics in combination with an ECVFL to adjust Gaussian minimum beam waist location and size for ideal zero propagation loss receive beam capture. The proposed low loss link can be beneficial to energy saving communication operations, for example, in portable devices and data centres where max- 
imum data rates must be sent with minimal power usage. A visible laser smart wireless link is demonstrated for a variable $1 \mathrm{~m}$ to $4 \mathrm{~m}$ link distance. The spatially conditioned link achieved a $<7 \%$ optical propagation loss over the $4 \mathrm{~m}$ range and showed a 53\% improvement over an unconditioned laser link. The smart link has the ability to provide a wide area search beam for search and hand-shake operations with other receiving $\mathrm{T} / \mathrm{R}$ units, easing alignment procedures and beam blocking instances. Future work relates to smart link demonstrations with high data rates.

\section{ACKN OWLEDGMENTS}

The authors thank Dr. Frank Perez and Nuonics, Inc. for the DMD-based beam imager instrument equipment support.

\section{References}

[1] F. R. Gfeller, and U. H. Bapst, "Wireless in-house data communication via diffuse infrared radiation" Proc. IEEE 67, 1474-1486 (1979).

[2] C. S. Yen, and R. D. Crawford, "The Use of Directed Optical Beams in Wireless Computer Communication" Proc. of IEEE Global Comm. Conf. 1985, New Orleans, U.S.A., pp.1181-1184 (1985).

[3] Y. Nakata, J. Kashio, T. Kojima, and T. Noguchi, “In-house wireless communication system using infrared radiation" in Proceedings to the Seventh International Conference on Computer Communications, 333-338 (IEEE, Sydney, 1985).

[4] A. Mahdy, and J. S. Deogun, "Wireless optical communications: A survey" in Proceedings to IEEE Wireless Communications and Networking Conference 4, 2399-2404 (IEEE, Atlanta, 2004).

[5] R. J. Green, H. Joshi, M. D. Higgins, and M .S. Leeson, "Recent developments in indoor optical wireless systems" IET Commun. 2, 3-10 (2008).

[6] H. Elgala, R. Meshleh, and H. Haas, "Indoor Optical Wireless Communication: Potential and State-of-the-Art" IEEE Commun. Mag., 56-62 (2011).

[7] N. A. Riza, "Switchboard in the sky:freespace optics platform for communications and processing" IEEE LEOS Ann. Mtg. 2, 211-212 (1998).

[8] N. A. Riza, "Reconfigurable optical wireless" in Proceedings to IEEE Lasers and Electro- Optics Society 1999 12th Annual Meeting, 70-71 (IEEE, San Francisco, 1999).

[9] N. A. Riza, and S. A. Khan, "Ultra-low loss laser communications technique using smart beamforming optics" Opt. Commun. 257, 225-246 (2006).

[10] J. Grubor, S. Randel, K.-D. Langer, and J. W. Walewski "Bandwidthefficient indoor optical wireless communications with white lightemitting diodes" in Proceedings to the 6th International Symposium on Communication Systems, Networks and Digital Signal Processing, 165-169 (IEEE, Graz, 2008).

[11] Y. Tanaka, T. Komine, S. Haruyama, and M. Nakagawa, "Indoor Visible Light Data Transmission System Utilizing White LED Lights" IEICE T. Commun. E86-B, 2440-2454 (2003).

[12] J. Vučić, C. Kottke, S. Nerreter, K.-D. Langer, and J. W. Walewski, “ $513 \mathrm{Mbit} / \mathrm{s}$ Visible Light Communications Link Based on DMTModulation of a White LED" J. Lightwave Technol. 28, 3512-3518 (2010).
[13] J. Alwan, Eye safety and wireless optical networks (WONs) (AirFiber Inc., 2001).

[14] J. T. Verdeyen, Laser Electronics (Prentice Hall, 3rd ed., New Jersey, 1994).

[15] N. A. Riza and F. N. Ghauri, "Super resolution hybrid analog-digital optical beam profiler using digital micromirror device" IEEE Photonic. Tech. L. 17, 1492-1494 (2005). 